\title{
Airwaves
}

\section{Outcome of influenza}

In this month's Thorax we publish two papers on outcomes of influenza. In the first paper, Fajardo-Dolci and colleagues report on clinical characteristics of 100 consecutive fatalities due to influenza A (H1N1) virus in Mexico which is regarded as the source of the recent pandemic. They discuss the disproportionate number of young adults who died due to H1N1 infection early in the outbreak and show that these patients had a high frequency of one or more chronic diseases on admission. In the accompanying editorial Dunning and Openshaw discuss the paper and point out that in Mexico access to anti-viral drugs was poor and fatalities were associated with high use of oral steroids. They warn that $\mathrm{H} 1 \mathrm{~N} 1$ may return in the coming winter and as immunity against evolving $\mathrm{H} 1 \mathrm{~N} 1$ strains is variable in the community, we must be prepared to respond with appropriate countermeasures to reduce fatalities. In the 2nd paper, Lee and colleagues report outcomes of adults hospitalised with severe seasonal influenza. The authors conclude that antiviral therapy for severe influenza is associated with reduced mortality and improved outcomes. They also emphasise that prompt case recogni-

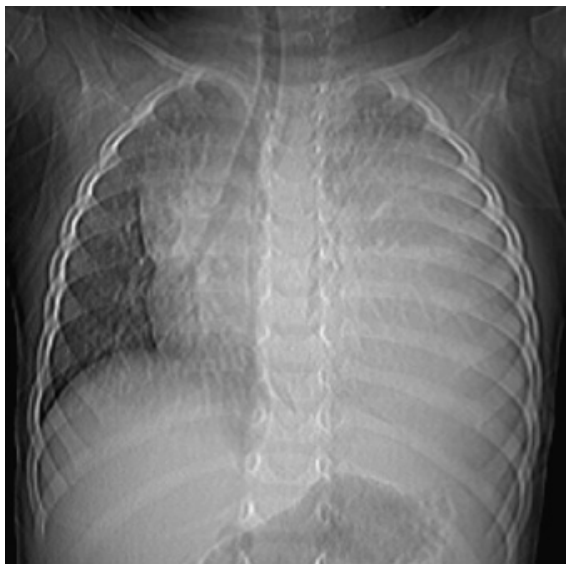

Chest radiography demonstrating opacification of the lower two-thirds of the left hemithorax. Part of the left upper lobe is still aerated. See Images in Thorax, page 555

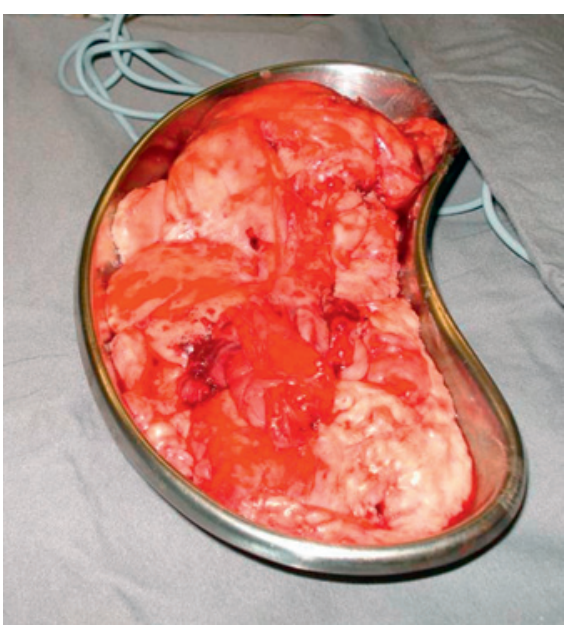

Homogeneous whitish tissue, removed during surgery, compatible with thymic hyperplasia. See Images in Thorax, page 555

tion and accurate diagnosis are essential so that timely therapeutic intervention can be considered. See pages 471, 505 and 510

\section{Arsenic exposure and respiratory symptoms}

As Parvez and colleagues point out in their paper, nearly 150 million people from Bangladesh and west Bengal continue to

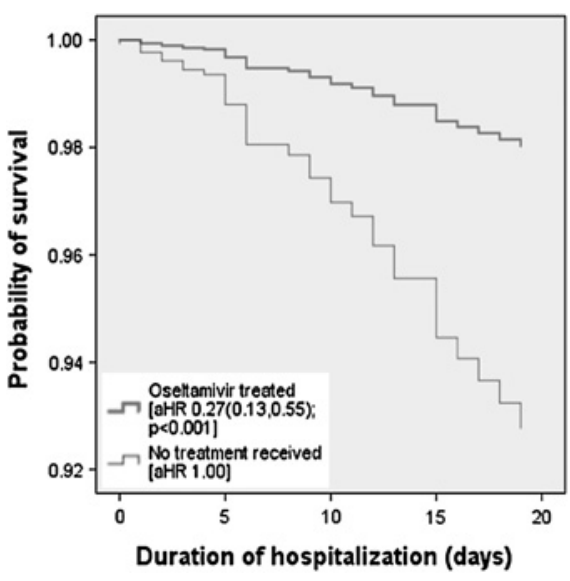

Survival of patients hospitalised with influenza and stratified by anti-viral therapy. See page 513 be exposed to arsenic that is a known carcinogen from drinking water. However there is little information on the effect of arsenic on respiratory symptoms. In a prospective cohort study, the authors found a dose-response relationship between arsenic exposure and symptoms of respiratory disease that was evident in the low to moderate dose range. Thus these results suggest that a considerable part of the population is at risk of developing lung disease. See page $\mathbf{5 2 8}$

\section{Women are more susceptible to COPD}

Smoking and COPD among women is increasing and there have been suggestions that women are more susceptible to the effects of cigarette smoke. In this issue, Sorheim and colleagues show that female gender is associated with lung function reduction and more severe disease in subjects with COPD with early onset disease or low smoking exposure. The results suggested that women are more susceptible to the effects of smoking but the interactions between gender, lung function and the environment are complex and more work is required to understand the mechanisms involved. See page 480

\section{Effects of restrictive spirometric patterns}

It is known that restrictive patterns obtained on spirometry can lead to increased morbidity and mortality. In this issue Guerra and colleagues explore further this association in the Tuscon (TESAOD) study and show that $12 \%$ of the population had restrictive spirometry. They also report that $38 \%$ of these subjects with restrictive spirometry developed airway obstruction on follow-up and the remainder had considerable respiratory symptoms and were at higher risk of life-threatening comorbidities. In the accompanying editorial Mannino provides a historical perspective on the natural history of lung disease and suggests it is time for a new paradigm for chronic lower respiratory diseases. See page 469 and 499 